\title{
Pseudo-Kaposi Sarcoma Associated with Borrelia burgdorferi Infection and Seropositivity for p17, p19, p31, p39, VIsE and p83 Proteins \\ B Tas ${ }^{1}$, A Caglar $^{2}$
}

\begin{abstract}
Pseudo-Kaposi's sarcoma also known as acroangiodermatitis (AAD) is a benign, uncommon angioproliferative skin disease which affects lower limbs. It is thought to be a reaction pattern to underlying chronic venous failure. We present a 71-year-old woman with unhealing wounds on her right limb, which was bitten by ticks two years ago. Except for the other laboratory examinations which were within the normal limits or negative, the patient's borrelia IgM was negative and IgG was positive. Based on the clinicopathological evidence, the patient was diagnosed with AAD. The lesions were thought to be associated with chronic borrelia infection, and they were treated with doxycycline. All of the lesions healed three months after the initiation of treatment, leaving atrophic scars. The patient was presented because no case of AAD associated with borrelia infection has been reported in literature.
\end{abstract}

Keywords: Acroangiodermatitis, Borrelia burgdorferi, lyme disease, molecular mimicry

From: ${ }^{1}$ Department of Dermatology, Bagcilar Research and Training Hospital ${ }^{2}$ Bagcilar Research and Training Hospital Department of Pathology.

Correspondence: Dr B Tas, Atakoy 7-8. Kisım, Martı Sitesi, 14/105, postal code: 34156, Bakirkoy/Istanbul. Fax: (90) 212-4404000. E-mail: betulavc@yahoo.com 


\section{INTRODUCTION}

Pseudo-Kaposi's sarcoma or AAD, which is a benign vascular dermatosis, resembles Kaposi sarcoma both clinically and histopathologically, and can be confused with it and other malignancies. It is most commonly associated with chronic venous insufficiency (Mali type). Other associated conditions are chronic arteriovenous malformations (Stewart-Bluefarb type), arteriovenous shunts in dialysis patients, first pregnancy, extremity paralysis and amputation stumps $(1,2)$. On the other hand, Lyme disease (LD) is an infectious disease caused by spirochetes called "Borrelia burgdorferi (Bb)", as a result of a tick bite. Besides the classical manifestations (erythema migrans, lymphadenosis benigna cutis, acrodermatitis chronica atrophicans), some other skin diseases associated with borrelia infections have been reported recently, including morphea, lichen sclerosus, systemic sclerosis, cutaneous sarcoidosis, necrobiosis lipoidica, necrobiotic xanthogranuloma, eosinophilic fasciitis, atrophoderma of Pasini and Pierini, pseudolymphoma, septal panniculitis resembling erythema nodosum, progressive facial hemiatrophy of Parry-Romberg, sclerodermatous porphyria cutanea tarda, interstitial granulomatous dermatitis and granuloma annulare $(3,4,5)$.

\section{CASE REPORT}

A 71-year-old woman was admitted to our dermatology clinic complaining of unhealing wounds on her right shin, which first appeared 6 months ago. According to personal history, the shin of the patient was bitten by ticks two years ago, and the current wounds appeared on the previous tick bite sites as small papules, and subsequently increased in size and turned into ulcers. She had not received any treatment for these tick bites. The other history was unremarkable. She was seen by a few physicans for leg wounds, and received some treatments of topical steroids and oral antihistamines, but the lesions did not improve. In the 
dermatological examination, there were violaceous $5.5 \times 3.5 \mathrm{~cm}, 2 \times 2 \mathrm{~cm}$ and $1.5 \times 1.5 \mathrm{~cm}$ in diameter plaques on the right shin. In the middle of the plaques, there was an ulcerous area consisting of impetiginous and necrotic crusts, a clean-based ulcer, and a superficial crust, respectively (Figure 1a). The lesions were symptomless. Physical examinations of the patient was normal. Wound cultures remained sterile. In the laboratory parameters, in addition to routine hematological and biochemical examinations, and urinalysisis, C-reactive protein, cryoglobulins, serological examinations of syphilis, anti-HIV antibodies, markers of hepatitis, rheumatoid factor, antinuclear antibody, TSH, FT3, FT4 and tumor markers were normal or within the normal limits. Doppler ultrasonography of both lower extremities showed grade-2 venous insufficiencies, equally. A chest X-ray and abdominal ultrasonography were normal, and mantoux test was negative. In the ELISA tests, borrelia IgM was negative (3.63 RU /ml), and borrelia $\mathrm{IgG}$ was positive $(59.25 \mathrm{RU} / \mathrm{ml})$ (cut-off values:16 RU/ml). WB test was performed to confirm the results, and $\mathrm{IgG}$ was strong positive against p17, p19, p31 and p83, and positive against VIsE and p39 antigens of Bb (Figure 2). In the cutaneous histopathology, a moderate epidermal hyperkeratosis and acanthosis, increased vascular endothelial proliferations without atypia, slight erythrocyte extravasation and congestion, and a small number of perivascular lymphocytes in papillary dermis were seen (Figure 3a,b). Proliferated endothelial cells of the vessels were positive for CD34 immunohistochemistry, while perivascular cells were not (Figure 3c). With the clinicopathological evidence, the lesions were diagnosed as AAD of Mali. The lesions were thought to be associated with chronic borrelia infection, and the patient was treated with oral doxycycline for 30 days $(200 \mathrm{mg} /$ day $)$. No additional treatment was applied to the lesions. The lesions gradually decreased from the third week. No adverse effect was observed during the treatment. All the lesions healed completely two months after the initiation of treatment 
leaving atrophic scars. The patient is in the sixth month after recovery, and still under follow up. No recurrence has been observed so far.

\section{DísCUSSION}

AAD was first described by Mali et al. in 1965 (1). Reported cases are usually associated with underlying venous failure, but it can be seen in chronic arteriovenous malformations, first gestation, and arteriovenous shunts in patients with chronic kidney failure $(1,2)$. However, a few spontaneous cases, and some cases related to homozygous activated protein $\mathrm{C}$ resistance, and hereditary coagulation defects such as a mutation in the prothrombin gene have been reported. Mali type usually occurs in elderly patients as mostly bilaterally venous stasis dermatitis (1). Although exact pathogenesis is unknown, a chronic tissue hypoxia, resulting from neovascularization and fibroblast proliferation following an increased capillary pressure in cases of chronic venous stasis has been suggested (2). The clinical manifestations of AAD can be highly variable, consisting of multiple, purpuric macules, reddish-brown or violaceous papules, plaques, nodules or ulcers, located on the lower limb, especially on the ankles, feet, toes or shins $(1,2,6,7)$. Because we did not detect any of related causes aside from the slight venous failure, our patient diagnosed with AAD of Mali. In view of available laboratory investigations of our patient, we did not find any mentioned causes which can be associated with the etiopathogenesis, except for history of tick bites, strong immunoreactivity for four IgG bands and weak immunorectivity for two IgG bands. It is not yet known whether borrelia antigens involved in etiopathogenesis of AAD. However, Aguero-Rosenfelt et al. stated that some $\mathrm{Bb}$ proteins such as flagellin $(41 \mathrm{kDa})$, FLaA $(37 \mathrm{kDa})$, OpsA( $31 \mathrm{kDa})$, OpsB (34kDa), OpsC (21 to $25 \mathrm{kDa})$, BmpA (39kDa), VIsE were highly immunogenic, and certain flagellin epitopes also cross-reacted with antigens found in mammalian tissues such 
as neural tissues, synovium, and myocardial muscle $(8,9)$. Dai et al. indicated that the flagellin cross-reacts with human axons and the protein can play a role in LD through molecular mimicry (10). One of the highly immunogenic proteins of $\mathrm{Bb}$ is $\mathrm{p} 83$ (also called p93/p100) which is similar to eukaryotic cell structures. Rössler et al. speculated that p83 might be involved in the mechanisms of LD by mimicking these structures (11). Autoreactivity is based on antigenic cross-reactivity between similar epitopes of $\mathrm{Bb}$ and the human host, especially situated on the heat shock proteins (Hsps). Many Hsps of Bb such as p60, p66, p43, p72, p24, p35, p28 have been identified $(11,12)$. Moreover, it has been stated that lyme arthritis might be the result of a persistent $\mathrm{Bb}$ infection in which borrelial DNA and antigens were retained in the patient, and these DNA products might be responsible for induction of an autoimmunity due to a T-cell-receptor epitope mimicry (13). Anti-borrelia IgM rises 2-4 weeks after the tick bite and may persist at high levels while anti-borrelia IgG might not rise for many years after the tick bite $(14,15)$. Both IgM and IgG may persist for many years even after successful treatment of LD. Patients with late manifestations of LD usually have a high concentration of antibodies in the tests and have numerous immunoreactive bands in IgG immunoblots (13). It has been postulated that antibodies against some immunodominant borrelia proteins such as p41, p25 (OspC), p37 (or p35), p45, p39 might be detected at the early-stage of LD whereas some others such as p31(OspA), p34(OspB), p28 and p30 might be detected at the late-stage of LD. Aguere et al. have stated that the antibodies against p39 were more often detected during the convalescence phases than acute phases $(8,9)$. On the other hand, p17 (also called Ops17) is one of the immunogenic proteins of $\mathrm{Bb}$ and is associated with binding of $\mathrm{Bb}$ to the host collagenassociated proteoglican decorin (9). When we took into account the amount of the time elapsed after the tick bites (18 months), strong positivity for P83, P17, P19, and P31 proteins, weak positivity for VIsE and P39 proteins, and negativity for p25 and IgM antibodies, the 
stage of our patient's disease was compatible with the late LD. We think that an antigenic cross-reactivity due to the T-cell type epitope mimicry might have developed between dermal vessels of the patient and these $\mathrm{Bb}$ proteins. Moreover, due to the presence of strongest positivity for $\mathrm{p} 17$, we also think that it might have acted as trigger of the autoimmunity in vascular collagen. Histopathology of AAD shows thick-walled neovascularization, and proliferated and plump endothelial cells of pre-existing vessels in the upper dermis. Additionally, extravasated erythrocytes, small trombi in small vessels, a few perivascular lymphocytic infiltrations and hemosiderin deposition can be detected $(1,2,7)$. Immunohistochemically, staining for CD34 was positive in only vascular endothelial cells, but not in the perivascular cells. With the staining feature, AAD can be differentiated from Kaposi sarcoma in which both cell types are positive for CD34 $(1,2)$. Other differential diagnoses include other vascular tumors, vasculitis, lichen simplex chronicus, lichen planus, basal-cell carcinoma (6), erythema induratum, and deep mycoses (1). The treatment of AAD depends on the underlying disturbances (6). On the other hand, LD should be treated at allstages of the disease, and doxycycline is the mainstay of therapy of cutaneous borrelia infections. The duration of treatment should be at least four weeks in late-stages (13), as was done by us. To the best of our knowledge, the presented case is the first example of AAD associated with $\mathrm{Bb}$ infection.

\section{ACKNOWLEDGEMENT}

None

Conflicts of interest: The authors declare that they did not have any conflicts of interest 


\section{REFERENCES}

1. Singh SK, Manchanda K. Acroangiodermatitis (Pseudo-Kaposi sarcoma). Indian Dermatol Online J 2014;5:323-5.

2. Mehta AA, Pereira RR, Nayak CS, Dhurat RS. Acroangiodermatitis of mali: a rare vascular phenomenon. Indian J Dermatol Venereol Leprol 2010;76:553-6.

3. Müllegger RR, Glatz M. Skin manifestations of lyme borreliosis: diagnosis and management. Am J Clin Dermatol 2008;9:355-68.

4. Eisendle K, Zelger B. The expanding spectrum of cutaneous borreliosis. G Ital Dermatol Venereol 2009;144:157-71.

5. Fernandez-Flores A, Ruzic-Sabljic E. Granuloma annulare displaying pseudorosettes in Borelia infection. Acta Dermatovenereol Alp Panonica Adriat $2008 ; 17: 171-6$.

6. Pimentel MI, Cuzzi T, Azeredo-Coutinho RB, Vasconcellos Éde C, Benzi TS, Carvalho LM. Acroangiodermatitis (pseudo-Kaposi sarcoma): a rarely-recognized condition. A case on the plantar aspect of the foot associated with chronic venous insufficiency. An Bras Dermatol 2011;86(4 Suppl 1):S13-6.

7. Bernardes Filho F, Martins G, Nery JA, de Andrade CV, Kac BK. Acroangiodermatitis (pseudo-Kaposi's sarcoma) in an HIV sero-positive patient with syphilis and hepatitis $\mathrm{C}$ virus coinfection: clinical and dermatopathological features. An Bras Dermatol 2014;89:792-5.

8. Chandra A, Wormser GP, Marques AR, Latov N, Alaedini A. Anti-Borrelia burgdorferi antibody profile in post-Lyme disease syndrome. Clin Vaccine Immunol 2011;18:767-71.

9. Aguero-Rosenfeld ME, Wang G, Schwartz I, Wormser GP. Diagnosis of lyme . Clin Microbiol Rev 2005;18:484-509. 
10. Dai Z, Lackland H, Stein S et al. Molecular mimicry in Lyme disease: monoclonal H9724 to B. burgdorferi flagellin specifically detects chaperonin-HSP60. Biochim Biophys Acta 1993; 1181:97-100.

11. Rössler D, Eiffert H, Jauris-Heipke $\mathrm{S}$ et al. Molecular and immunological characterization of the p83/100 protein of various Borrelia burgdorferi sensu lato strains. Med Microbiol Immunol 1995;184:23-32.

12. Carreiro MM, Laux DC, Nelson DR. Characterization of the heat shock response and identification of heat shock protein antigens of Borrelia burgdorferi. Infect Immun 1990;58:2186-91.

13. Gischick HJ, Morbach H, Tappe D. Treatment of Lyme borreliosis. Arthritis Res Ther 2009;11: 258 .

14. Cutler SJ, Wright DJ. Predictive value of serology in diagnosing Lyme borreliosis. J Clin Pathol 1994;47: 344-49.

15. Gajović O, Todorović Z, Nesić L, Lazić Z. Lyme borreliosis--diagnostic difficulties in interpreting serological results. Med Pregl 2010;63:839-43. [ Article in Serbian] 


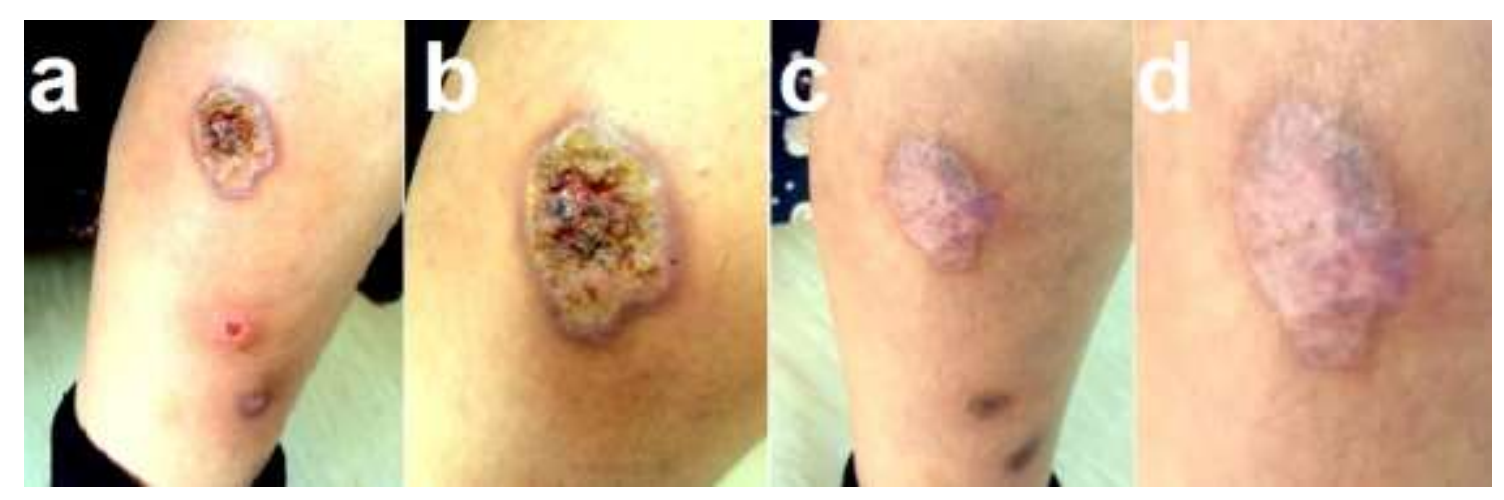

Fig 1. Clinical presentation of ulcerous plaques surrounded by thin varicous veins on the right shin, before the treatment. $(a, b)$. Postinflammatory atrophic scars after the treatment $(\mathrm{c}, \mathrm{b})$.
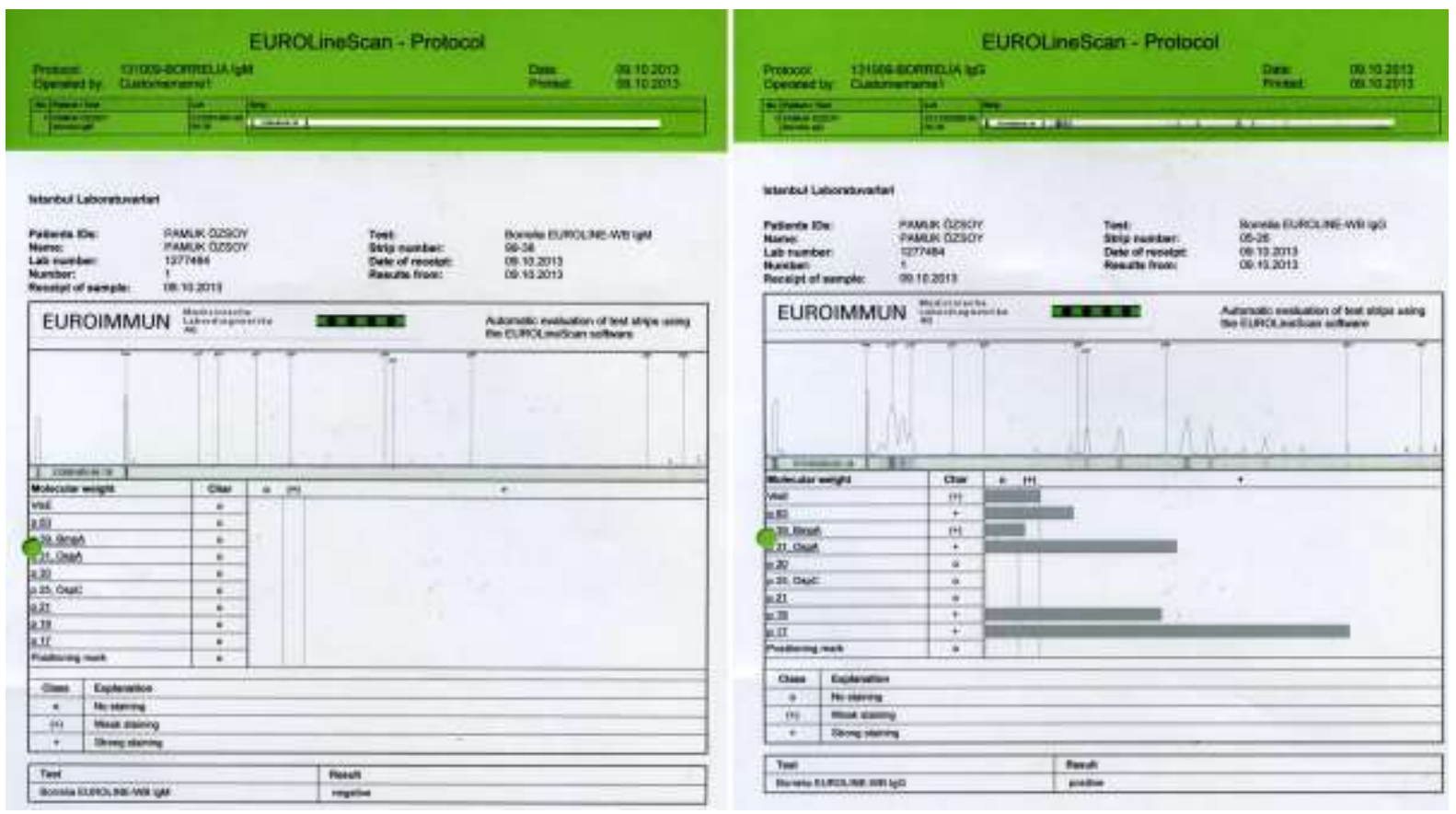

Fig 2. Negativity of anti-borrelia IgM, and positive IgG immunoblots of the patient, in the western immunoblotting tests. 


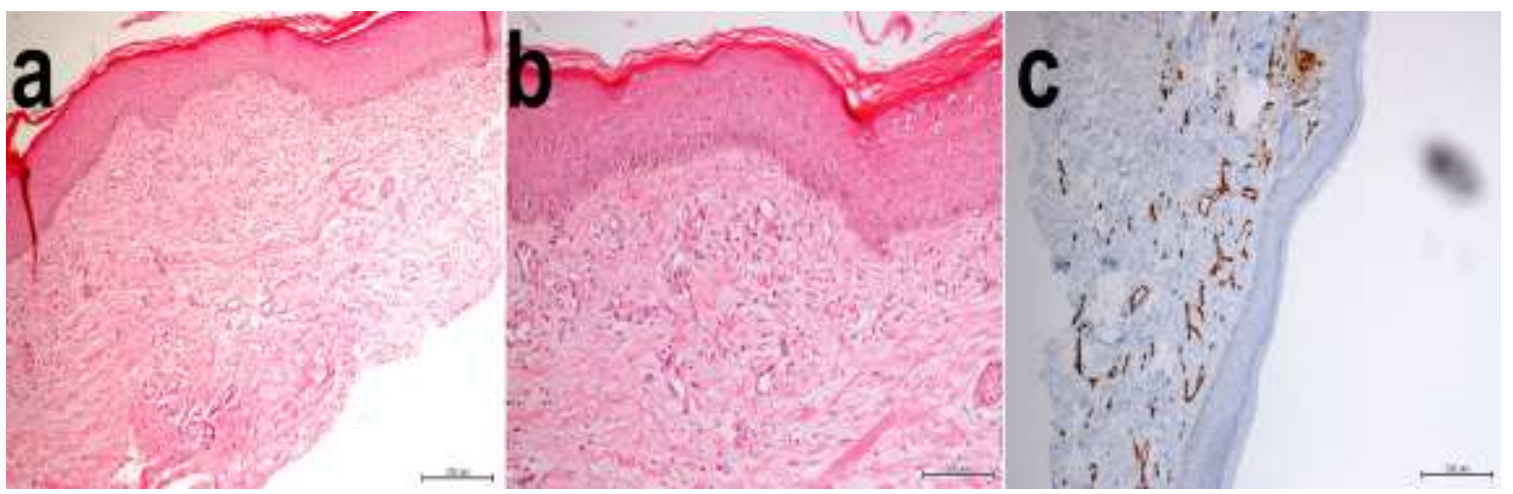

Fig 3. A slight epidermal hyperkeratosis and acanthosis, and proliferation of endothelium in small vessels, a few extravazated erythrocytes around the vessels along with a small number of perivascular lymphocytes in the papillary dermis (a. HEX100, b. HEX200). Immunopositivity for anti-CD34 antibody restricted to the vascular endothelium (c. CD34X100). 